\title{
Accurate analysis of harmonic Hall voltage measurement for spin-orbit torques
}

\author{
Seok Jin Yun ${ }^{1}$, Eun-Sang Park ${ }^{2}$, Kyung-Jin Lee ${ }^{1,2}$ and Sang Ho Lim ${ }^{1}$ \\ An accurate method is developed to extract the spin-orbit (SO) effective fields by analyzing harmonic Hall voltage measurements \\ and deriving detailed analytical equations that consider both the $z$ component of the applied magnetic field and the second- \\ order perpendicular magnetic anisotropy. The method is tested by analyzing the results of a macrospin simulation. The SO \\ effective fields that are extracted from the analysis are consistent with the input SO effective fields that are used in the \\ macrospin simulation over the entire range of the polar magnetization angle and for a wide range (0-2) of the ratio of the planar \\ to the anomalous Hall voltage considered in this study. The accuracy of the proposed method is demonstrated via a systematic \\ study that involves comparing its results with those of a conventional analytical method.
}

NPG Asia Materials (2017) 9, e449; doi:10.1038/am.2017.200; published online 17 November 2017

\section{INTRODUCTION}

In-plane currents in a nonmagnetic (NM)/ferromagnetic (FM) bilayer nanostructure can generate a torque due to the spin-orbit (SO) coupling, which is known as the SO torque (SOT). SOT is sufficient for reversing the magnetization in the FM layer. ${ }^{1}$ Numerous studies have been conducted to identify the principal mechanism of the SOT as either the spin Hall effect (SHE) in the $\mathrm{NM} \mathrm{layer}^{2-4}$ or the interfacial SO coupling-frequently referred to as the Rashba effectat the NM/FM interface. ${ }^{5-12}$ In a system in which the NM/FM interface is perpendicular to the $z$ axis and the in-plane currents flow along the $x$ axis, spin currents that are polarized along the $y$ axis are generated in the system based on the SHE induced by a bulk SO coupling in the NM layer. The spin currents are injected into the adjacent FM layer, which causes torque to be transferred to the magnetization of the FM layer. The SHE-induced SOT generates a strong damping-like torque $\left(\boldsymbol{T}_{\mathrm{DL}} \propto \boldsymbol{m} \times \boldsymbol{m} \times y\right)$ and a weak field-like torque $\left(\boldsymbol{T}_{\mathrm{FL}} \propto \boldsymbol{m} \times y\right){ }^{13,14}$ Theoretically, the strength of the SHEinduced SOT is known to be independent of the magnetization direction of the FM layer. For an interfacial SO coupling-induced SOT, spins that are polarized along the $y$ axis accumulate due to the broken inversion symmetry at the NM/FM interface. Direct exchange coupling between the magnetization of the FM layer and the accumulated spins generates a strong $\boldsymbol{T}_{\mathrm{FL}}$ but a weak $\boldsymbol{T}_{\mathrm{DL}} \cdot{ }^{7,15-18}$ Unlike the strength of the SHE-induced SOT, the interfacial SO coupling-induced SOT is known to depend on the magnetization direction of the FM layer. ${ }^{19-21}$ In these two cases, both the SHE and the interfacial SO coupling qualitatively induce the same torque on the FM layer. To identify the dominant mechanism of the SOT, a quantitative analysis of the values of $\boldsymbol{T}_{\mathrm{DL}}$ and $\boldsymbol{T}_{\mathrm{FL}}$ over an extensive range of magnetization angles is required. ${ }^{19-21}$
The harmonic Hall voltage measurement method is a useful approach for quantifying the effective fields of $T_{\mathrm{DL}}$ and $T_{\mathrm{FL}}$ that originate from the SOT. ${ }^{22}$ This method is particularly suitable for identifying the angular dependence of the SOT that acts on the FM layer with perpendicular magnetization. ${ }^{19,20}$ Several corrections are required for an accurate analysis of the measured results, including the measured results for the planar Hall effect (PHE), ${ }^{20,23}$ the out-of-plane component of the external magnetic field ${ }^{20}$ and the anomalous Nernst effect. $^{20,24}$ In the harmonic Hall voltage measurement, the second harmonic voltage $\left(V^{2 \omega}\right)$ consists of two major components: anomalous Hall voltage and planar Hall voltage (denoted as $V_{\mathrm{AHE}}$ and $V_{\mathrm{PHE}}$, respectively). ${ }^{20,23}$ When an external magnetic field $\left(\boldsymbol{H}_{\text {ext }}\right)$ is applied along the longitudinal $(x)$ direction, the $V^{2 \omega}$ values from the AHE and PHE are proportional to $T_{\mathrm{DL}}$ and $T_{\mathrm{FL}}$, respectively. Under a transverse (y) $\boldsymbol{H}_{\mathrm{ext}}$, however, these values are proportional to $\boldsymbol{T}_{\mathrm{FL}}$ and $\boldsymbol{T}_{\mathrm{DL}}$, respectively. This necessitates the use of an analytical solution that is based on Cramer's rule to separate $\boldsymbol{T}_{\mathrm{FL}}$ and $\boldsymbol{T}_{\mathrm{DL}}{ }^{23}$ This analytical solution has been successfully applied to only a system in which $V_{\mathrm{PHE}}<V_{\mathrm{AHE}}$. For a system in which $V_{\mathrm{PHE}}>V_{\mathrm{AHE}}$, such as the W/ $\mathrm{CoFeB} / \mathrm{MgO}$ trilayer structure, ${ }^{25}$ a divergence occurs in the solution, which makes it extremely difficult to analyze the measurement results.

This problem can be overcome by incorporating some necessary corrections into the analysis of the measurement results, including measurement results for the out-of-plane component of $\boldsymbol{H}_{\text {ext }}$. As coherent magnetization rotation is an important requirement in the analysis of harmonic Hall voltage measurement results, $\boldsymbol{H}_{\mathrm{ext}}$ is usually applied along the direction tilted slightly $\left(4^{\circ}-15^{\circ}\right)$ from the basal plane $\left(x-y\right.$ plane) ${ }^{20}$ In this condition, the $z$ component of $\boldsymbol{H}_{\text {ext }}$ has a nonzero value, although it has been neglected to simplify the analysis. This assumption is reasonable in the low- $\boldsymbol{H}_{\text {ext }}$ range, where the

${ }^{1}$ Department of Materials Science and Engineering, Korea University, Seoul, Korea and ${ }^{2}$ KU-KIST Graduate School of Converging Science and Technology, Korea University, Seoul, Korea

Correspondence: Professor SH Lim, Department of Materials Science and Engineering, Korea University, 145 Anam-ro, Seongbuk-gu, Seoul 02841, Korea.

E-mail: sangholim@korea.ac.kr

Received 21 March 2017; revised 31 August 2017; accepted 25 September 2017 
magnetization direction is close to the $z$ axis and the perpendicular magnetic anisotropy (PMA) field is consequently dominant over the $z$ component of $\boldsymbol{H}_{\text {ext }}{ }^{23}$ The simplifying assumption, however, is not valid in the high- $\boldsymbol{H}_{\text {ext }}$ range, where the magnetization direction deviates considerably from the $z$ axis, with a resultant reduction in the PMA field. As a result, the PMA field loses its dominance over the $z$ component of $\boldsymbol{H}_{\text {ext }}$. Several attempts have been made to include the $z$ component of $\boldsymbol{H}_{\text {ext }}$, which is obtained by repetitively solving equilibrium torque equations until the desired convergence is achieved (the recursive method). ${ }^{19,20}$ However, this method is complicated, and it has not been validated for systems with $V_{\mathrm{PHE}}>V_{\mathrm{AHE}}$. Unwanted voltages, which originate from thermoelectric effects such as the anomalous Nernst effect, should be eliminated from the harmonic signals. Although several methods have been proposed for this purpose, ${ }^{20,24}$ erasing all artificial signals remains difficult. Another important issue that has not been considered is the inclusion of the second-order PMA in an analysis of the harmonic Hall voltage measurement results. The inclusion of the second-order PMA is considered important because many PMA materials exhibit secondorder PMA, whose strength is comparable to that of the first-order PMA in many cases. ${ }^{26,27}$

In this study, two corrections-one for the $z$ component of $\boldsymbol{H}_{\mathrm{ext}}$ and the other for the second-order PMA-are considered in the analysis of the harmonic Hall voltage measurement results. All related analytical equations are described. Both conventional and refined analytical methods are used to analyze the results of the macrospin simulation, which mimics the harmonic Hall voltage measurement by numerically solving the Landau-Lifshitz-Gilbert equation. ${ }^{28,29}$ The accuracy of these two analytical methods is tested by comparing the input SO effective fields for the macrospin simulation with the input SO effective fields calculated by analytical methods. To test the refined analytical method, systems are examined over a wide ratio $R$, which is defined as $V_{\mathrm{PHE}} / V_{\mathrm{AHE}}$. A similar comparative study that analyzes the experimental results of harmonic Hall voltage measurements for a Pt/ $\mathrm{Co} / \mathrm{MgO}$ structure was also performed.

\section{MATERIALS AND METHODS}

\section{Analytical solutions for conventional approach}

When an in-plane AC current with frequency $\omega\left(I_{\mathrm{AC}}=I_{0} \sin \omega t\right)$ is applied to an NM/FM bilayer structure, the angle between the $z$ axis and the magnetization of the FM layer $\left(\theta_{M}\right)$ and the angle between the $x$ axis and the orthographic projection of the magnetization on the $x-y$ plane $\left(\varphi_{M}\right)$ oscillate as $\theta_{M}(t)=\theta_{M}{ }^{\circ}$ $+\Delta \theta_{M} \sin \omega t$ and $\varphi_{M}(t)=\varphi_{M}{ }^{\circ}+\Delta \varphi_{M} \sin \omega t$. Here the superscript ${ }^{\circ}$ and the symbol $\Delta$ denote the value in the absence of $I_{\mathrm{AC}}$ and the amplitude of the related angles, respectively. The total energy equation for the NM/FM bilayer structure can be expressed as follows:

$$
\begin{aligned}
& E_{\mathrm{tot}}=-K_{1}^{\mathrm{eff}} \cos ^{2} \theta_{M}-K_{2} \cos ^{4} \theta_{M}-M_{\mathrm{S}} \boldsymbol{m} \cdot\left(\boldsymbol{H}_{\mathrm{ext}}+\Delta \boldsymbol{H}\right), \\
& \boldsymbol{m} \equiv\left(\sin \theta_{M} \cos \varphi_{M}, \sin \theta_{M} \sin \varphi_{M}, \cos \theta_{M}\right), \\
& \boldsymbol{H}_{\mathrm{ext}} \equiv H_{\mathrm{ext}}\left(\sin \theta_{H} \cos \varphi_{H}, \sin \theta_{H} \sin \varphi_{H}, \cos \theta_{H}\right), \\
& \Delta \boldsymbol{H} \equiv \Delta H_{\mathrm{DL}} \boldsymbol{m} \times \hat{y}-\Delta H_{\mathrm{FL}} \hat{y} .
\end{aligned}
$$

Here $K_{1}$ eff is the effective first-order PMA energy density that considers the demagnetizing term, that is, $K_{1}{ }^{\text {eff }}=K_{1}-N_{\mathrm{d}} M_{\mathrm{S}}^{2} / 2\left(K_{1}, N_{\mathrm{d}}\right.$ and $M_{\mathrm{S}}$ are the firstorder PMA energy density, demagnetizing factor and saturation magnetization, respectively). ${ }^{30} K_{2}$ is the second-order PMA energy density. ${ }^{30} m$ is the unit vector of magnetization. The effective magnetic field $(\Delta \boldsymbol{H})$ induced by the maximum value of the in-plane AC current $\left(I_{0}\right)$ is composed of the dampinglike effective field $\left(\Delta H_{\mathrm{DL}}\right)$ and the field-like effective field $\left(\Delta H_{\mathrm{FL}}\right) . \theta_{H}$ and $\varphi_{H}$ are the polar angle and azimuthal angle of $\boldsymbol{H}_{\mathrm{ext}}$, respectively. Given that the inplane anisotropy is negligibly small over the PMA field, $\varphi_{M}{ }^{\circ}$ is assumed to be identical to $\varphi_{H}$. The values of $\Delta \theta_{M}$ and $\Delta \varphi_{M}$ can be analytically expressed as follows (refer to Supplementary Equations (S1)-(S13) for a detailed derivation):

$$
\begin{aligned}
\Delta \theta_{M} & =-\frac{\Delta H_{\mathrm{DL}} \cos \varphi_{H}+\Delta H_{\mathrm{FL}} \cos \theta_{M}^{\mathrm{o}} \sin \varphi_{H}}{H_{\mathrm{K}}^{\mathrm{eff}} \cos 2 \theta_{M}^{\mathrm{o}}-H_{K, 2} \sin \theta_{M}^{\mathrm{o}} \sin 3 \theta_{M}^{\mathrm{o}}+H_{\mathrm{ext}} \cos \left(\theta_{M}^{\mathrm{o}}-\theta_{H}\right)}, \\
\Delta \varphi_{M} & =\frac{\Delta H_{\mathrm{DL}} \cos \theta_{M}^{\mathrm{o}} \sin \varphi_{H}-\Delta H_{\mathrm{FL}} \cos \varphi_{H}}{H_{\mathrm{ext}} \sin \theta_{H}} .
\end{aligned}
$$

Here $H_{\mathrm{K}}{ }^{\text {eff }}$ and $H_{\mathrm{K} 1}{ }^{\text {eff }}$ are the effective PMA field and the effective first-order PMA field, respectively, and $H_{\mathrm{K}, 2}$ is the second-order PMA field. These parameters are defined as follows: $H_{\mathrm{K}}{ }^{\text {eff }} \equiv H_{\mathrm{K}, 1}{ }^{\text {eff }}+H_{\mathrm{K}, 2} ; \quad H_{\mathrm{K}, 1}{ }^{\text {eff }} \equiv 2 K_{1}{ }^{\text {eff }} / M_{\mathrm{S}}$; $H_{\mathrm{K}, 2} \equiv 4 K_{2} / M_{\mathrm{S}}$. Note that Equations (5) and (6) are identical to the analytical expressions derived by Hayashi et al. ${ }^{23}$ when $H_{\mathrm{K}, 2}=0$. If the values of $\Delta \theta_{M}$ and $\Delta \varphi_{M}$ are sufficiently small, the components of the $m$ vector can be approximated in the form $m(t)=m^{\circ}+(2 \Delta m) \sin \omega t$ :

$$
\begin{aligned}
& m_{x} \approx \sin \theta_{M}^{\circ} \cos \varphi_{H} \\
& \quad+\left(\Delta \theta_{M} \cos \theta_{M}^{\mathrm{o}} \cos \varphi_{H}-\Delta \varphi_{M} \sin \theta_{M}^{\mathrm{o}} \sin \varphi_{H}\right) \sin \omega t, \\
& m_{y} \approx \sin \theta_{M}^{\mathrm{o}} \sin \varphi_{H} \\
& \quad+\left(\Delta \theta_{M} \cos \theta_{M}^{\circ} \sin \varphi_{H}+\Delta \varphi_{M} \sin \theta_{M}^{\mathrm{o}} \cos \varphi_{H}\right) \sin \omega t, \\
& m_{z} \approx \cos \theta_{M}^{\mathrm{o}}-\Delta \theta_{M} \sin \theta_{M}^{\mathrm{o}} \sin \omega t .
\end{aligned}
$$

Both the anomalous Hall voltage and planar Hall voltage contribute to the measured Hall voltage: $V_{\mathrm{H}}=I_{\mathrm{AC}} R_{\mathrm{H}}=I_{\mathrm{AC}} R_{\mathrm{AHE}} m_{z}+I_{\mathrm{AC}} R_{\mathrm{PHE}} m_{x} m_{y}{ }^{20,31,32}$ Here $R_{\mathrm{AHE}}$ and $R_{\mathrm{PHE}}$ are the anomalous and planar Hall resistance, respectively. With the application of $I_{\mathrm{AC}}$, the $m$ values oscillate as given in Equations (7)-(9) with the resultant expressions for Hall voltages as follows:

$$
\begin{aligned}
& V_{\mathrm{H}}=\left(V_{\mathrm{AHE}} m_{z}+V_{\mathrm{PHE}} m_{x} m_{y}\right) \sin \omega t=V^{1 \omega} \sin \omega t-V^{2 \omega} \cos 2 \omega t, \\
& V_{x}^{1 \omega}=V_{y}^{1 \omega}=V_{\mathrm{AHE}} \cos \theta_{M}^{\mathrm{o}}, \\
& V_{x}^{2 \omega}=-\frac{V_{\mathrm{AHE}}}{2}\left[\sin \theta_{M}^{\mathrm{o}} \Delta \theta_{M}-R \sin ^{2} \theta_{M}^{\mathrm{o}} \Delta \varphi_{M}\right], \\
& V_{y}^{2 \omega}=-\frac{V_{\mathrm{AHE}}}{2}\left[\sin \theta_{M}^{\mathrm{o}} \Delta \theta_{M}+R \sin ^{2} \theta_{M}^{\mathrm{o}} \Delta \varphi_{M}\right] .
\end{aligned}
$$

Here the following relations exist: $V_{\mathrm{AHE}}=I_{0} R_{\mathrm{AHE}}$ and $V_{\mathrm{PHE}}=I_{0} R_{\mathrm{PHE}}$. The subscripts $x$ and $y$ indicate that the harmonic Hall voltages are measured at $\varphi_{H}=0^{\circ}$ and $\varphi_{H}=90^{\circ}$, respectively. The first harmonic Hall voltage $\left(V^{1 \omega}\right)$ contains information about the $\theta_{M}{ }^{\circ}$ value, and the second harmonic Hall voltage $\left(V^{2 \omega}\right)$ contains information about the $\Delta \theta_{M}$ and $\Delta \varphi_{M}$ values. The conventional analytical solution considers only the case in which the magnetization direction has slightly deviated from the $z$ axis $\left(\theta_{M}{ }^{\circ} \approx 0^{\circ}\right)$. In this case, the $z$ component of $\boldsymbol{H}_{\text {ext }}$ is negligibly small over the PMA field along the same direction $\left(H_{\text {ext }} \cos \theta_{H} \ll H_{\mathrm{K}}{ }^{\text {eff }} \cos \theta_{M}{ }^{\circ}\right)$, and therefore, the assumption of $\sin \theta_{M^{\circ}}{ }^{\circ}=H_{\text {ext }} / H_{\mathrm{K}}$ eff in the conventional solution is reasonable. ${ }^{8,23,33}$ Note that the contribution due to $H_{\mathrm{K}, 2}$ is also negligible at $\theta_{M}{ }^{\circ} \approx 0^{\circ}$ (Equation (5)). With this assumption, $V^{1 \omega}$ and $V^{2 \omega}$ can be rewritten as follows:

$$
\begin{aligned}
& V_{x}^{1 \omega}=V_{y}^{1 \omega}=V_{\mathrm{AHE}} \sqrt{1-\left(\frac{H_{\mathrm{ext}}}{H_{\mathrm{K}}^{\mathrm{eff}}}\right)^{2}}, \\
& V_{x}^{2 \omega}=\frac{V_{\mathrm{AHE}} H_{\mathrm{ext}}}{2\left(H_{\mathrm{K}}^{\mathrm{eff}}\right)^{2}}\left[\frac{\Delta H_{\mathrm{DL}}}{1-\left(H_{\mathrm{ext}} / H_{\mathrm{K}}^{\mathrm{eff}}\right)^{2}}-R \Delta H_{\mathrm{FL}}\right], \\
& V_{y}^{2 \omega}=\frac{V_{\mathrm{AHE}} H_{\mathrm{ext}}}{2\left(H_{\mathrm{K}}^{\mathrm{eff}}\right)^{2}} \sqrt{1-\left(\frac{H_{\mathrm{ext}}}{H_{\mathrm{K}}^{\mathrm{eff}}}\right)^{2}}\left[\frac{\Delta H_{\mathrm{FL}}}{1-\left(H_{\mathrm{ext}} / H_{\mathrm{K}}^{\mathrm{eff}}\right)^{2}}-R \Delta H_{\mathrm{DL}}\right] .
\end{aligned}
$$

The second harmonic Hall voltages, as given in Equations (15) and (16), are composed of two terms that contain $\Delta H_{\mathrm{DL}}$ and $\Delta H_{\mathrm{FL}}$. When the $R$ ratio is negligibly small, the values of $\Delta H_{\mathrm{DL}}$ and $\Delta H_{\mathrm{FL}}$ can be obtained using the 
following $T$ ratios:

$$
\begin{aligned}
& T_{x} \equiv-\frac{2 V_{x}^{2 \omega}}{\left(\partial V_{x}^{1 \omega} / \partial H_{\mathrm{ext}}\right)}\left(\frac{V_{x}^{1 \omega}}{V_{\mathrm{AHE}}}\right)=A_{0} \Delta H_{\mathrm{DL}}-B_{0} \Delta H_{\mathrm{FL}}, \\
& T_{y} \equiv \frac{2 V_{y}^{2 \omega}}{\left(\partial V_{y}^{1 \omega} / \partial H_{\mathrm{ext}}\right)}=B_{0} \Delta H_{\mathrm{DL}}-A_{0} \Delta H_{\mathrm{FL}} \\
& A_{0} \equiv 1, B_{0} \equiv R\left(1-\left(\frac{H_{\mathrm{ext}}}{H_{\mathrm{K}}^{\mathrm{eff}}}\right)^{2}\right) .
\end{aligned}
$$

At $R=0$, the values of $T_{x}$ and $T_{y}$ are identical to the values of $\Delta H_{\mathrm{DL}}$ and $-\Delta H_{\mathrm{FL}}$, respectively. ${ }^{8}$ When the $R$ ratio becomes comparatively large, $T_{x}$ and $T_{y}$ should be corrected using Cramer's rule. ${ }^{23}$

$$
\left(\begin{array}{c}
\Delta H_{\mathrm{DL}} \\
\Delta H_{\mathrm{FL}}
\end{array}\right)=\frac{1}{B_{0}^{2}-A_{0}^{2}}\left(\begin{array}{cc}
-A_{0} & B_{0} \\
-B_{0} & A_{0}
\end{array}\right)\left(\begin{array}{c}
T_{x} \\
T_{y}
\end{array}\right) .
$$

In Equation (20), $\Delta H_{\mathrm{DL}}$ and $\Delta H_{\mathrm{FL}}$ can be calculated if the determinant $B_{0}^{2}-A_{0}^{2}$ is not 0 . If $B_{0}^{2}-A_{0}^{2}$ is 0 , it is not possible to obtain the individual values of $\Delta H_{\mathrm{DL}}$ and $\Delta H_{\mathrm{FL}}$; it is only possible to obtain the relation $T_{x}=T_{y}=\Delta H_{\mathrm{DL}}-\Delta H_{\mathrm{FL}}$ (Equations (17)-(19)).

\section{Analytical solutions for refined approach}

The assumption of $H_{\text {ext }} \cos \theta_{H} \ll H_{\mathrm{K}}{ }^{\text {eff }} \cos \theta_{M}{ }^{\circ}$ is not valid at high $H_{\text {ext }}$ values. In this case, the $\theta_{H}$ value is not negligible, and $\Delta \theta_{M}$ and $\Delta \varphi_{M}$ (Equations (5) and (6)) must be substituted into Equations (12) and (13) to obtain the expression for $V^{2 \omega}$ :

$$
\begin{aligned}
& V_{x}^{2 \omega}=\frac{V_{\mathrm{AHE}}}{2}\left[A_{1} \Delta H_{\mathrm{DL}}-B_{1} \Delta H_{\mathrm{FL}}\right], \\
& V_{y}^{2 \omega}=-\frac{V_{\mathrm{AHE}} \cos \theta_{M}^{\mathrm{o}}}{2}\left[B_{1} \Delta H_{\mathrm{DL}}-A_{1} \Delta H_{\mathrm{FL}}\right], \\
& A_{1} \equiv \frac{\sin \theta_{M}^{\mathrm{o}}}{H_{\mathrm{K}}^{\mathrm{eff}} \cos 2 \theta_{M}^{\mathrm{o}}-H_{K, 2} \sin \theta_{M}^{\mathrm{o}} \sin 3 \theta_{M}^{\mathrm{o}}+H_{\mathrm{ext}} \cos \left(\theta_{M}^{\mathrm{o}}-\theta_{H}\right)}, \\
& B_{1} \equiv \frac{R \sin ^{2} \theta_{M}^{\mathrm{o}}}{H_{\mathrm{ext}} \sin \theta_{H}} .
\end{aligned}
$$

Considering that $V_{x}{ }^{1 \omega}=V_{y}{ }^{1 \omega}=V_{\mathrm{AHE}} \cos \theta_{M}{ }^{\circ}$ (Equation (11)), the $G$ ratios, which correspond to the $T$ ratios in the conventional approach, can be defined as follows:

$$
\begin{aligned}
& G_{x} \equiv \frac{2 V_{x}^{2 \omega}}{V_{\mathrm{AHE}}}=A_{1} \Delta H_{\mathrm{DL}}-B_{1} \Delta H_{\mathrm{FL}}, \\
& G_{y} \equiv-\frac{2 V_{y}^{2 \omega}}{V_{y}^{1 \omega}}=B_{1} \Delta H_{\mathrm{DL}}-A_{1} \Delta H_{\mathrm{FL}} .
\end{aligned}
$$

Similar to the conventional analytical equations, the refined equations also need to be solved using Cramer's rule (as given in Equation (20)).

$$
\left(\begin{array}{c}
\Delta H_{\mathrm{DL}} \\
\Delta H_{\mathrm{FL}}
\end{array}\right)=\frac{1}{B_{1}^{2}-A_{1}^{2}}\left(\begin{array}{cc}
-A_{1} & B_{1} \\
-B_{1} & A_{1}
\end{array}\right)\left(\begin{array}{c}
G_{x} \\
G_{y}
\end{array}\right) .
$$

\section{RESULTS}

\section{Conventional analysis}

The conventional analytical method is used to analyze the results of the macrospin simulation. Figure $1 \mathrm{a}$ and $\mathrm{b}$ shows the results for $V^{1 \omega}$ as a function of $H_{\text {ext }}$ in two different systems: (a) $H_{\mathrm{K}, 1}$ eff $=5 \mathrm{kOe}$ and $H_{\mathrm{K}, 2}=0 \mathrm{kOe}$; and (b) $H_{\mathrm{K}, 1}{ }^{\text {eff }}=5 \mathrm{kOe}$ and $H_{\mathrm{K}, 2}=-1 \mathrm{kOe}$. Two sets of results are shown in Figure 1a and b: the first set is obtained from the macrospin simulation (squares), and the second set is obtained from Equation (14), which is based on the conventional assumption of $\sin \theta_{M}{ }^{\circ}=H_{\text {ext }} / H_{\mathrm{K}}{ }^{\text {eff }}$ (dashed lines). For the macrospin simulation, the following parameters are applied: $\Delta H_{\mathrm{DL}}=-50$ Oe; $\Delta H_{\mathrm{FL}}=-100$ Oe; $\theta_{H}=86^{\circ}$; and $V_{\mathrm{AHE}}=1 \mathrm{mV}$. Refer to Supplementary Figure $\mathrm{S} 1$ for a detailed description of the macrospin simulation. Agreement between the results obtained from the macrospin simulation and the results obtained from Equation (14) based on the conventional analytical method, which is the focus of this study, is only obtained in the low$H_{\text {ext }}$ range. In the high- $H_{\text {ext }}$ range, the deviation is very large, which indicates the limited validity of conventional solutions.

Figure $1 \mathrm{c}$ and $\mathrm{d}$ shows the analytical results for $B_{0}^{2}-A_{0}{ }^{2}$ calculated from Equation (19) as a function of $H_{\text {ext }}$ at two different $R$ values of 0.3 (red lines) and 1.75 (blue lines) $\left(R=V_{\mathrm{PHE}} / V_{\mathrm{AHE}}\right)$. The results in Figure 1c pertain to the system with $H_{\mathrm{K}, 1}$ eff $=5 \mathrm{kOe}$ and $H_{\mathrm{K}, 2}=0$, and the results in Figure 1d pertain to the system with $H_{\mathrm{K}, 1}{ }^{\text {eff }}=5 \mathrm{kOe}$ and $H_{\mathrm{K}, 2}=-1 \mathrm{kOe}$. The $H_{\mathrm{K}}{ }^{\text {eff }}$ values for both systems are also indicated in the figures. The detailed equation for $B_{0}^{2}-A_{0}^{2}$ is rewritten for clarity:

$$
B_{0}{ }^{2}-A_{0}{ }^{2}=R^{2}\left(1-\left(\frac{H_{\mathrm{ext}}}{H_{\mathrm{K}}^{\mathrm{eff}}}\right)^{2}\right)^{2}-1 .
$$

Recalling that $H_{\mathrm{ext}} / H_{\mathrm{K}}{ }^{\text {eff }}$ is approximated with $\sin \theta_{M}{ }^{\circ}$, we note that the results for $B_{0}^{2}-A_{0}^{2}$ at $H_{\text {ext }}>H_{\mathrm{K}}{ }^{\text {eff }}$ have no physical meaning. According to Equation (28), the $B_{0}{ }^{2}-A_{0}^{2}$ value decreases from $R^{2}-1$ to -1 as the $H_{\text {ext }}$ value increases from 0 to $H_{\mathrm{K}}{ }^{\text {eff }}$. The determinant $B_{0}{ }^{2}-A_{0}{ }^{2}$ always has a negative value at $R<1$. At $R \geqslant 1$, however, the determinant can have both positive values and negative values over the $H_{\text {ext }}$ range of $0-H_{\mathrm{K}}$ eff, which indicates the occurrence of a crossover $\left(B_{0}^{2}-A_{0}^{2}=0\right)$ at a certain $H_{\text {ext }}$ value. This feature is visible in the results shown in Figure $1 \mathrm{c}$ and $\mathrm{d}$. In both systems, that is, with $H_{\mathrm{K}, 2}=0$ and $-1 \mathrm{kOe}$, the $B_{0}{ }^{2}-A_{0}^{2}$ value is always negative at $R=0.3$; at $R=1.75$, it initially has a positive value, after which it passes through 0 and then has a negative value. The crossovers occur at 3.3 and $2.6 \mathrm{kOe}$ for the systems with $H_{\mathrm{K}, 2}=0 \mathrm{kOe}$ and $H_{\mathrm{K}, 2}=-1 \mathrm{kOe}$, respectively. Recalling that $T_{x}=T_{y}=\Delta H_{\mathrm{DL}}-\Delta H_{\mathrm{FL}}$ when the determinant is 0 , an $H_{\text {ext }}$ value should exist at $T_{x}=T_{y}$ when $R>1$.

Figure $2 \mathrm{a}-\mathrm{f}$ shows the results for $V_{x}^{2 \omega}$ and $V_{y}{ }^{2 \omega}$ (a and b) and $T_{x}$ and $T_{y}$ (c and d) as functions of $H_{\text {ext }}$ and the results for $\Delta H_{\mathrm{DL}}$ and $\Delta H_{\mathrm{FL}}$ (e and $\mathrm{f}$ ) as functions of $\theta_{M}{ }^{\circ}$ for two different systems, that is, with $H_{\mathrm{K}, 2}=0$ (solid lines) and $H_{\mathrm{K}, 2}=-1 \mathrm{kOe}$ (dashed lines). The left (Figure $2 \mathrm{a}, \mathrm{c}$ and e) and right panels (Figure $2 \mathrm{~b}, \mathrm{~d}$ and $\mathrm{f}$ ) show the results for $R=0.3$ and 1.75 , respectively. The results for $V^{2 \omega}$ were obtained by the macrospin simulation, and the results for $T_{x}$ and $T_{y}$ (Equations (17) and (18)) and $\Delta H_{\mathrm{DL}}$ and $\Delta H_{\mathrm{FL}}$ (Equation (20)) were analytically calculated using the simulation results. As shown in Figure $2 \mathrm{a}$ and $\mathrm{b}$, the sign of $V_{x}^{2 \omega}$ for a small $R$ value of 0.3 is negative, whereas the sign of $V_{x}^{2 \omega}$ for a large $R$ value of 1.75 is positive. Of the two major contributions of $V_{\mathrm{AHE}}$ and $V_{\mathrm{PHE}}$ to $V^{2 \omega}$, the sign of the former is negative, but that of the latter is positive. Equations (15) and (16) predict this behavior (the opposite signs of $V_{\mathrm{AHE}}$ and $V_{\mathrm{PHE}}$ ) and explain that the $V_{y}^{2 \omega}$ value for $R=1.75$ is higher than that for $R=0.3$.

The results for $V_{x}^{2 \omega}$ and $V_{y}^{2 \omega}$ and their variation with $R$ have a critical effect on $T_{x}$ and $T_{y}$. At $R=0.3$, the signs of $T_{x}$ and $T_{y}$ are opposite because the signs of $V_{x}^{2 \omega}$ and $V_{y}^{2 \omega}$ are the same, indicating that there are no $H_{\mathrm{ext}}$ values at which $T_{x}=T_{y}$ in both systems, that is, for $H_{\mathrm{K}, 2}=0 \mathrm{kOe}$ and $H_{\mathrm{K}, 2}=-1 \mathrm{kOe}$ (Figure 2c). These results are consistent with the results for $B_{0}^{2}-A_{0}^{2}$ (Figure $1 \mathrm{c}$ and d). The values of $T_{x}$ and $T_{y}$ are the same at a specific $H_{\text {ext }}$ value, at which $B_{0}{ }^{2}-A_{0}{ }^{2}=0$ (Equation (19)). For $R=1.75$, the signs of $T_{x}$ and $T_{y}$ are the same because the signs of $V_{x}{ }^{2 \omega}$ and $V_{y}{ }^{2 \omega}$ are opposite (Figure $2 \mathrm{~d}$ ). There are $H_{\text {ext }}$ values for which $T_{\mathrm{x}}=T_{y}$ exist in both systems, that is, with $H_{\mathrm{K}, 2}=0 \mathrm{kOe}$ and $H_{\mathrm{K}, 2}=-1 \mathrm{kOe}$. The positions, however, differ from those for which $B_{0}{ }^{2}-A_{0}{ }^{2}=0$. The $H_{\text {ext }}$ values in 


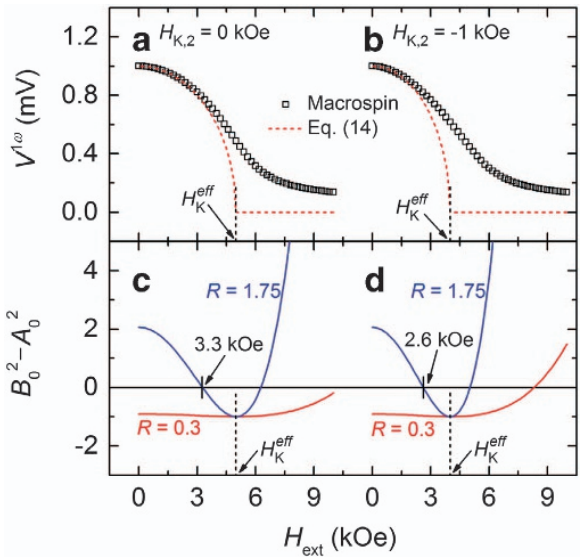

Figure 1 Results for $V^{1 \omega}(\mathbf{a}, \mathbf{b})$ and $B_{0}^{2}-A_{0}^{2}(\mathbf{c}, \mathbf{d})$ as functions of $H_{\text {ext }}$ for systems with $H_{k, 1}$ eff $=5 \mathrm{kOe}$ and $H_{k, 2}=0 \mathrm{kOe}$ (left panels; a, c) and $H_{\mathrm{k}, 1}{ }^{\text {eff }}=5 \mathrm{kOe}$ and $H_{\mathrm{k}, 2}=-1 \mathrm{kOe}$ (right panels; b, d). For the macrospin simulation, the following parameters are used: $\Delta H_{\mathrm{DL}}=-50 \mathrm{Oe}$; $\Delta H_{\mathrm{FL}}=-100 \mathrm{Oe} ; \theta_{H}=86^{\circ}$; and $V_{\mathrm{AHE}}=1 \mathrm{mV}$. In a, b, two sets of results are shown: one set of results from the macrospin simulation (squares) and one set from Equation (14), which is based on the conventional analytical method (dashed lines). In c, $\mathbf{d}$, two sets of results, both of which are obtained by the conventional analytical method, are shown for $R=0.3$ (red lines) and $R=1.75$ (blue lines).

the former case are 3.6 and $3.8 \mathrm{kOe}$ for the systems with $H_{\mathrm{K}, 2}=0$ and $H_{\mathrm{K}, 2}=-1 \mathrm{kOe}$, respectively, whereas those in the latter case are 3.3 and $2.6 \mathrm{kOe}$, respectively. These deviations occur because the determinant poorly reflects the behavior of the first harmonic.

The inappropriate determinant, that is, $B_{0}^{2}-A_{0}^{2}$, causes large errors in the SO effective fields, as shown in Figure $2 \mathrm{e}$ and $\mathrm{f}$. Recalling that the input $\mathrm{SO}$ effective fields are $\Delta H_{\mathrm{DL}}=-50 \mathrm{Oe}$ and $\Delta H_{\mathrm{FL}}=-100 \mathrm{Oe}$, we consider the results at $R=0.3$ (Figure 2e) to be reliable in the $\theta_{M}{ }^{\circ}$ range from $0^{\circ}$ to the angles that correspond to

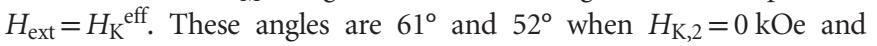
$H_{\mathrm{K}, 2}=-1 \mathrm{kOe}$, respectively. Beyond these two angles, which are indicated by the vertical and horizontal lines in Figure $2 \mathrm{e}$ and $\mathrm{f}$, respectively, the output $\mathrm{SO}$ effective fields start to deviate from the input values. The indicated regions end not at $90^{\circ}$ but at $\sim 82^{\circ}$ as the $\boldsymbol{m}$ vector is not completely aligned along the $x$ or $y$ axis, even for $H_{\text {ext }}=10 \mathrm{kOe}$ (Figure $1 \mathrm{a}$ and $\mathrm{b}$ ). The output SO effective fields reveal a divergence, which is physically meaningless at $\theta_{M}{ }^{\circ}=\sim 81^{\circ}$ $\left(H_{\mathrm{K}, 2}=0 \mathrm{kOe}\right)$ and $\sim 75^{\circ}\left(H_{\mathrm{K}, 2}=-1 \mathrm{kOe}\right)$. The deviations are very large at $R=1.75$ (Figure 2f). For the system with $H_{\mathrm{K}, 2}=0 \mathrm{kOe}$, divergence occurs even at $\sim 37^{\circ}$, which is not located in the region of physical insignificance (indicated by vertical lines). A similar behavior is observed for the system with $H_{\mathrm{K}, 2}=-1 \mathrm{kOe}$, where the divergence occurs at $\sim 32^{\circ}$. These divergences are attributed to the misallocation of the $H_{\text {ext }}$ value, at which $B_{0}^{2}-A_{0}^{2}=0$ (Figure 1c and d). The occurrence of the additional divergences significantly limits the reliability of the conventional analytical method for both systems, that is, with $H_{\mathrm{K}, 2}=0 \mathrm{kOe}$ and $H_{\mathrm{K}, 2}=-1 \mathrm{kOe}$, as shown in Figure $2 \mathrm{f}$.

\section{Refined analysis}

The main reason behind the unreliable results obtained from the conventional analysis is the determinant, which poorly describes the behavior of the first harmonic Hall voltage. For an accurate evaluation of the determinant, the refined analysis begins by determining the relation between $\theta_{M}{ }^{\circ}$ and $H_{\text {ext }}$, which can be obtained using the equation $\theta_{M}{ }^{\circ}=\cos ^{-1}\left(V^{1 \omega} / V_{\mathrm{AHE}}\right)$ or the total energy equation ${ }^{34}$
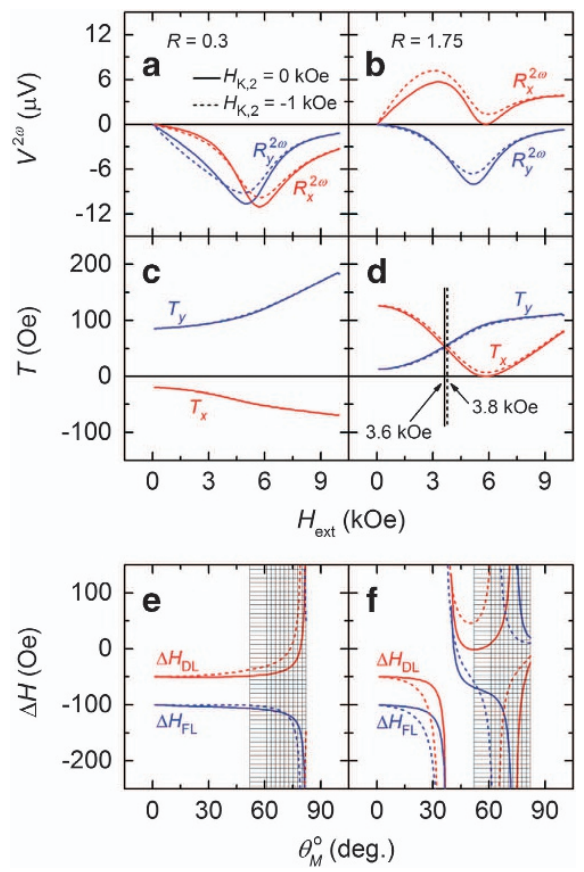

Figure 2 Results for $V_{x}^{2 \omega}$ and $V_{y}{ }^{2 \omega}(\mathbf{a}, \mathbf{b})$ and $T_{x}$ and $T_{y}(\mathbf{c}, \mathbf{d})$ as functions of $H_{\text {ext }}$ and those for $\Delta H_{\mathrm{DL}}$ and $\Delta H_{\mathrm{FL}}(\mathbf{e}, \mathbf{f})$ as functions of $\theta_{M}{ }^{\circ}$. The results for $V^{2 \omega}$ are obtained by a macrospin simulation, and those for $T_{x}, T_{y}, \Delta H_{\mathrm{DL}}$ and $\Delta H_{\mathrm{FL}}$ are obtained by analysis of the simulation results using a conventional analytical method. Results are obtained for the systems with $H_{\mathrm{K}, 2}=0 \mathrm{kOe}$ (solid lines) and $H_{\mathrm{K}, 2}=-1 \mathrm{kOe}$ (dashed lines). The left panels (a, c, e) show the results for $R=0.3$, whereas the right panels (b, d, f) show the results for $R=1.75$. In $\mathbf{e}, \mathbf{f}$, the regions filled with vertical lines and horizontal lines indicate the $\theta_{M}{ }^{\circ}$ range of no physical significance for the systems with $H_{\mathrm{K}, 2}=0$ and $H_{\mathrm{K}, 2}=-1 \mathrm{kOe}$, respectively.

(Equation (1)). The results for $V^{1 \omega}$ as a function of $H_{\text {ext }}$ (Figure 1a and b) can be applied to obtain the relation. Figure $3 \mathrm{a}$ and $\mathrm{b}$ shows the results for the determinant $B_{1}{ }^{2}-A_{1}{ }^{2}$ as a function of the $H_{\text {ext }}$ obtained from the refined analysis (Equations (23) and (24)) for the systems with $H_{\mathrm{K}, 2}=0 \mathrm{kOe}$ and $H_{\mathrm{K}, 2}=-1 \mathrm{kOe}$, respectively. The solid lines in Figure $3 \mathrm{a}$ and $\mathrm{b}$ indicate the results for $B_{1}{ }^{2}-A_{1}{ }^{2}$, as calculated using $H_{\mathrm{K}, 2}=0$ and $H_{\mathrm{K}, 2}=-1 \mathrm{kOe}$ from the macrospin simulation and the refined analysis, respectively. Using the relation between $\theta_{M}{ }^{\circ}$ and $\boldsymbol{H}_{\text {ext }}$ in the refined analysis enables the behavior of $V^{1 \omega}$ to be duly reflected in the determinant. At $R=1.75$, the $H_{\text {ext }}$ values for which $B_{1}{ }^{2}-A_{1}{ }^{2}=0$ are 3.6 and $3.8 \mathrm{kOe}$ for the systems with $H_{\mathrm{K}, 2}=0$ and $H_{\mathrm{K}, 2}=-1 \mathrm{kOe}$, respectively; these $H_{\text {ext }}$ values are identical to those obtained at $T_{x}=T_{y}$ (Figure 2d). To apply the behavior of $V^{1 \omega}$ to the determinant, the determinant $B_{1}{ }^{2}-A_{1}{ }^{2}$ needs to be calculated with a precise value of $H_{\mathrm{K}, 2}$, which was used in the macrospin simulation. To demonstrate the importance of the inclusion of $H_{\mathrm{K}, 2}$, the determinants were also calculated by disregarding $H_{\mathrm{K}, 2}$ (even though the actual $H_{\mathrm{K}, 2}$ value of the system is $-1 \mathrm{kOe}$ ); these results are also shown in Figure $3 \mathrm{~b}$ (dotted lines). The difference between the two cases of $R=0.3$ and 1.75 is very large, which indicates that $H_{\mathrm{K}, 2}$ should be considered in the analysis. At $R=1.75$, for example, the $H_{\text {ext }}$ value for which the determinant is 0 is misallocated from 3.8 to $3.2 \mathrm{kOe}$ when $H_{\mathrm{K}, 2}$ is disregarded; a new location that reveals a 0 value of the determinant emerges at $H_{\text {ext }}=9.0 \mathrm{kOe}$.

Figure $4 \mathrm{a}$ and $\mathrm{b}$ shows the results for $G_{x}$ and $G_{y}$, which correspond to $T_{x}$ and $T_{y}$ in the conventional analysis at $R=0.3$ and 1.75, respectively. The results for the systems with $H_{\mathrm{K}, 2}=0$ (solid lines) 


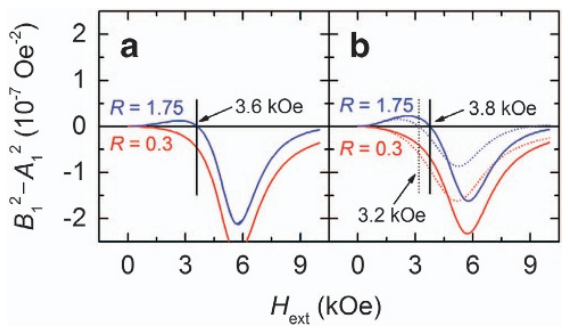

Figure 3 Refined analytical results for $B_{1}{ }^{2}-A_{1}{ }^{2}$ as a function of $H_{\text {ext }}$ for systems with (a) $H_{\mathrm{K}, 2}=0 \mathrm{kOe}$ and (b) $H_{\mathrm{K}, 2}=-1 \mathrm{kOe}$. Two sets of results, for $R=0.3$ (red lines) and $R=1.75$ (blue lines), are shown. In $\mathbf{b}$, the results calculated by disregarding $H_{\mathrm{K}, 2}$ (although it exists) are also shown (dotted lines).
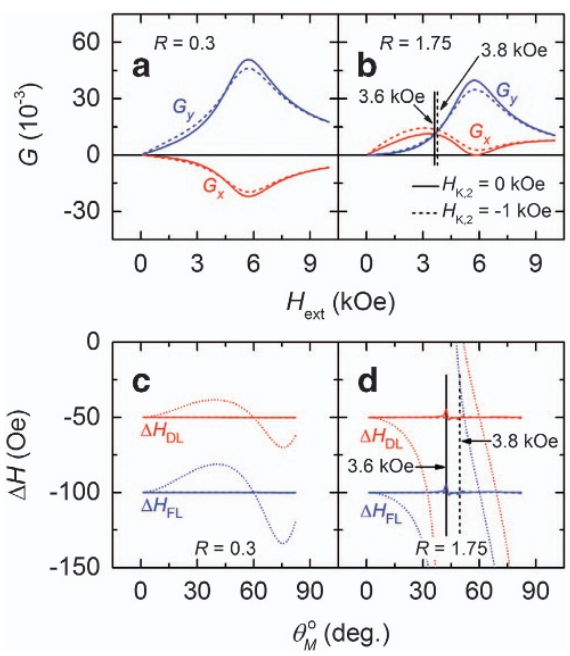

Figure 4 Refined analytical results for $G_{x}$ and $G_{y}$ as functions of $H_{\text {ext }}(\mathbf{a}, \mathbf{b})$ and refined analytical results for $\Delta H_{\mathrm{DL}}$ and $\Delta H_{\mathrm{FL}}$ as functions of $\theta_{M}{ }^{\circ}$ (c, d) for systems with $H_{k, 2}=0$ (solid lines) and $H_{k, 2}=-1 \mathrm{kOe}$ (dashed lines). The left panels (a, c) and right panels (b, d) show the results for $R=0.3$ and 1.75 , respectively. In $\mathbf{c}, \mathbf{d}$, the results calculated by disregarding $H_{\mathrm{k}, 2}$ (even though it exists) are also shown (dotted lines).

and $H_{\mathrm{K}, 2}=-1 \mathrm{kOe}$ (dashed lines) are shown. Note the $H_{\mathrm{ext}}$ values for which $G_{x}=G_{y}$ are 3.6 and $3.8 \mathrm{kOe}$ for the systems with $H_{\mathrm{K}, 2}=0 \mathrm{kOe}$ and $H_{\mathrm{K}, 2}=-1 \mathrm{kOe}$, respectively. These $H_{\mathrm{ext}}$ values are identical to the $H_{\text {ext }}$ values for which the determinant $B_{1}^{2}-A_{1}^{2}=0$ (Figure $3 \mathrm{a}$ and $\mathrm{b}$ ). This finding contradicts the case of the conventional analysis, where the $H_{\text {ext }}$ value for which the determinant is 0 differs substantially from the $H_{\text {ext }}$ value for which $T_{x}=T_{y}$ (Figures $1 \mathrm{c}$ and d, and $2 \mathrm{~d}$,). With the new set of results for the determinant and the $G$ ratios, the task of calculating the SO effective fields is straightforward; these results as a function of $\theta_{M}{ }^{\circ}$ are shown in Figure $4 \mathrm{c}$ and $\mathrm{d}$, for $R=0.3$ and 1.75, respectively. Two sets of results are shown: one set for the system with $H_{\mathrm{K}, 2}=0 \mathrm{kOe}$ (solid lines) and one set for the system with $H_{\mathrm{K}, 2}=-1 \mathrm{kOe}$ (dashed lines). As shown in Figure $4 \mathrm{c}$ and $\mathrm{d}$, in both systems, the calculated values of $\Delta H_{\mathrm{DL}}$ and $\Delta H_{\mathrm{FL}}$ are consistent with the input values for the macrospin simulation (over the entire $\theta_{M}{ }^{\circ}$ range of $0^{\circ}-\sim 82^{\circ}$ ); this finding demonstrates the reliability of the refined analysis. At $R=0.3$, the agreement between the two systems is perfect to such an extent that the solid lines for the $H_{\mathrm{K}, 2}=0 \mathrm{kOe}$ system completely overlap with the dashed lines for the $H_{\mathrm{K}, 2}=-1 \mathrm{kOe}$ system over the entire $\theta_{M}{ }^{\circ}$ range. A similar behavior is observed at $R=1.75$, with the difference that small peaks are observed at $\sim 43^{\circ}$ and

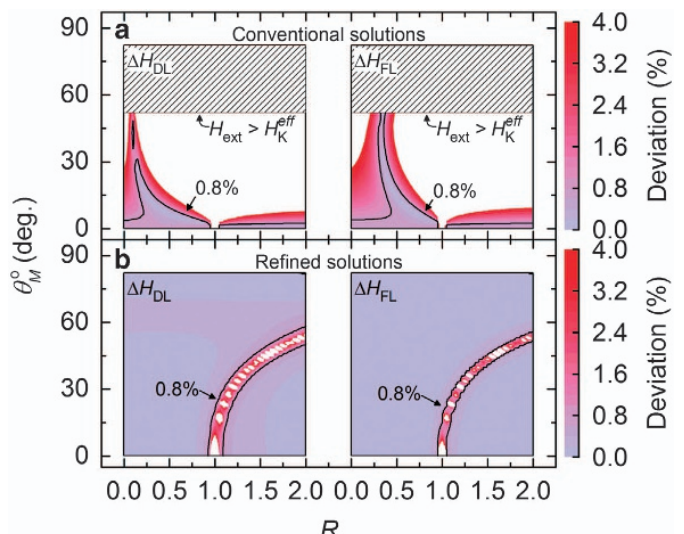

Figure 5 Contour plots that show deviation (in \%) from input values of $\Delta H_{\mathrm{DL}}$ (left panel) and $\Delta H_{\mathrm{FL}}$ (right panel) as a function of $\theta_{M}{ }^{\circ}$ and $R$. The results obtained from the conventional analytical method are shown in a, and the results obtained from the refined analytical method are shown in $\mathbf{b}$. All results are obtained for the system with $H_{k, 2}=-1 \mathrm{kOe}$. In a, the regions filled with inclined lines indicate the $\theta_{M}{ }^{\circ}$ range of no physical significance.
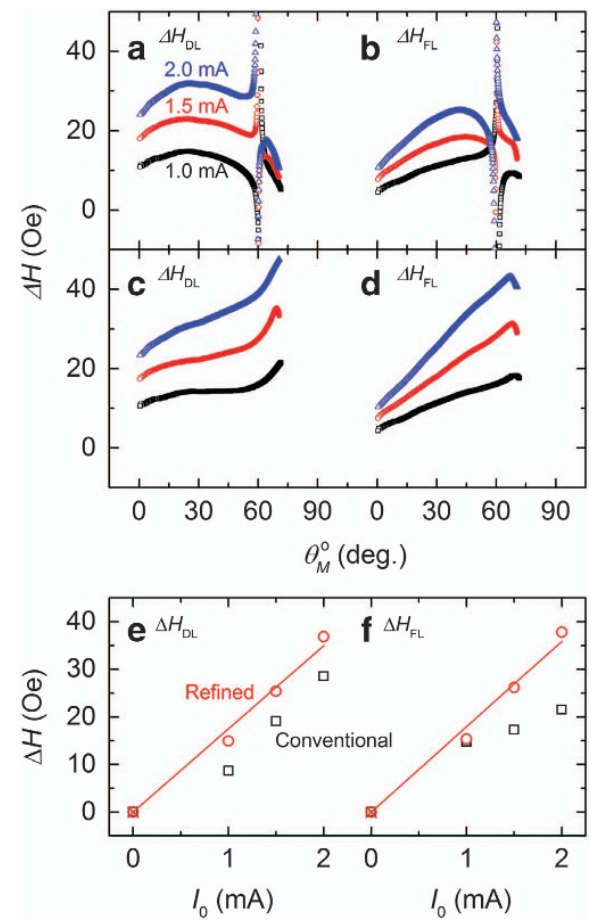

Figure 6 Results for $\Delta H_{\mathrm{DL}}(\mathbf{a}, \mathbf{c})$ and $\Delta H_{\mathrm{FL}}(\mathbf{b}, \mathbf{d})$ as functions of $\theta_{M}{ }^{\circ}$ at three different $I_{0}$ values of $1.0 \mathrm{~mA}$ (black squares), $1.5 \mathrm{~mA}$ (red circles) and $2.0 \mathrm{~mA}$ (blue triangles), which are calculated using conventional $(\mathbf{a}, \mathbf{b})$ and refined (c, d) analytical methods. The results for $\Delta H_{\mathrm{DL}}$ (e) and $\Delta H_{\mathrm{FL}}$ (f) obtained at a $\theta_{M}{ }^{\circ}$ value of $55^{\circ}$ as functions of $I_{0}$ calculated using the conventional (black squares) and refined (red circles) analytical methods. Lines in $\mathbf{e}, \mathbf{f}$ represent the least squares fit to the refined results.

$\sim 50^{\circ}$ for the systems with $H_{\mathrm{K}, 2}=0 \mathrm{kOe}$ and $H_{\mathrm{K}, 2}=-1 \mathrm{kOe}$, respectively, for which $B_{1}^{2}-A_{1}^{2}=0$.

In systems with both $H_{\mathrm{K}, 1}$ eff and $H_{\mathrm{K}, 2}$, the determinant $B_{1}{ }^{2}-A_{1}^{2}$ significantly differs if $H_{\mathrm{K}, 2}$ is disregarded (Figure 3b). A similar difference is expected for the calculated values of $\Delta H_{\mathrm{DL}}$ and $\Delta H_{\mathrm{FL}}$ (using Equation (27)), which are also shown in Figure $4 \mathrm{c}$ and $\mathrm{d}$ (dotted lines). For $R=0.3$, the absolute values of $\Delta H_{\mathrm{DL}}$ and $\Delta H_{\mathrm{FL}}$ are underestimated in the $\theta_{M}{ }^{\circ}$ range of $0^{\circ}-60^{\circ}$ and overestimated in the 
range of $60^{\circ}-\sim 82^{\circ}$. This finding is reasonable based on the $H_{\mathrm{K}, 2}$ term, which is proportional to $\sin \theta_{M}{ }^{\circ} \sin 3 \theta_{M}{ }^{\circ}$ (Equation (23)). For $R=1.75$, the differences are significant, with two divergences at $\sim 39^{\circ}$ and $\sim 80^{\circ}$ due to the misallocated $H_{\text {ext }}$ fields of 3.2 and $9.0 \mathrm{kOe}$, for which $B_{1}{ }^{2}-A_{1}{ }^{2}=0$ (Figure $3 \mathrm{~b}$ ). These results demonstrate that $H_{\mathrm{K}, 2}$ should not be neglected in the analysis of the harmonic measurement results in systems with both $H_{\mathrm{K}, 1}$ eff and $H_{\mathrm{K}, 2}$.

Comparison of conventional and refined analyses over wide $R$ range Two typical $R$ ratios of 0.3 and 1.75 have been considered. To test the refined analytical method over a wide $R$ range, a systematic study was conducted by varying the $R$ ratio from 0 to 2 in steps of 0.05 for the system with $H_{\mathrm{K}, 2}=-1 \mathrm{kOe}$. Figure $5 \mathrm{a}$ and b displays contour plots that show the deviation (in \%) from the input values of $\Delta H_{\mathrm{DL}}$ (left panels) and the input values of $\Delta H_{\mathrm{FL}}$ (right panels) as a function of $\theta_{M}{ }^{\circ}$ and $R$. The results calculated using the conventional analytical method are shown in Figure 5a, whereas the results calculated using the refined method are shown in Figure 5b. In the case of conventional solutions, the $\theta_{M}{ }^{\circ}$ range in which $H_{\text {ext }}>H_{\mathrm{K}}{ }^{\text {eff }}$ has no physical significance is indicated in Figure $5 \mathrm{a}$ as inclined lines. In Figure $5 \mathrm{a}$ and $\mathrm{b}$, the solid lines indicate a deviation of $0.8 \%$ and the white regions indicate a minimum deviation of $4 \%$. As shown in Figure $5 \mathrm{a}$, the conventional solutions are valid over very limited ranges of $\theta_{M}{ }^{\circ}$ and $R$. For example, the $R$ range in which the deviations are $<4 \%$ is $0.06-0.12$ for $\Delta H_{\mathrm{DL}}$ and $0.21-0.46$ for $\Delta H_{\mathrm{FL}}$ in the $\theta_{M}{ }^{\circ}$ range of $0^{\circ}-52^{\circ}$. For $R$ values higher than 1.1, the validity range is even more limited for both $\Delta H_{\mathrm{DL}}$ and $\Delta H_{\mathrm{FL}}$; specifically, the $\theta_{M}{ }^{\circ}$ values for which the deviations are $<4 \%$ are $4.5^{\circ}$ at $R=1.1$ and $7.9^{\circ}$ at $R=2.0$ for $\Delta H_{\mathrm{DL}}$ and $4.5^{\circ}$ at $R=1.1$ and $9.4^{\circ}$ at $R=2.0$ for $\Delta H_{\mathrm{FL}}$. In the intermediate $R$ range of $0.9-1.1$, the deviations are always larger than $4 \%$. The accuracy of the calculated results improves significantly with the use of the refined method, as shown in Figure 5b. With the $z$ component of $\boldsymbol{H}_{\text {ext }}$ considered in the refined analysis, no region has physical insignificance. The predictions made in the refined analysis are highly accurate. For $R<0.85$, the deviations are $<0.4 \%$ over the entire $\theta_{M}{ }^{\circ}$ range of $0^{\circ}-82^{\circ}$ for both $\Delta H_{\mathrm{DL}}$ and $\Delta H_{\mathrm{FL}}$. Even for $R>0.85$, the deviations are $<0.8 \%$ for both $\Delta H_{\mathrm{DL}}$ and $\Delta H_{\mathrm{FL}}$ over the entire region, except in the regions marked by solid lines, where the deviations are large due to the existence of divergences (zero determinants).

\section{Analysis of experimental results}

An additional test of the refined method was performed by analyzing the experimental results of the harmonic Hall voltage measurements for a stack with the following structure: Si substrate (wet-oxidized)/Ta $(5 \mathrm{~nm}) / \mathrm{Pt}(5 \mathrm{~nm}) / \mathrm{Co}(0.6 \mathrm{~nm}) / \mathrm{MgO}(2 \mathrm{~nm}) / \mathrm{Ta}(2 \mathrm{~nm})$. Refer to Supplementary Figures S2 and S3 for Hall bar dimensions. The results were obtained at three different $I_{0}$ values: $1.0 ; 1.5$; and $2.0 \mathrm{~mA}$. The magnetization direction was controlled by $H_{\text {ext }}$, which was swept from +90 to -90 kOe with two different directions: $\theta_{H}=85^{\circ}$ and $\varphi_{H}=0^{\circ}$; and $\theta_{H}=85^{\circ}$ and $\varphi_{H}=90^{\circ}$. The values of $H_{\mathrm{K}, 1}$ eff and $H_{\mathrm{K}, 2}$, which were extracted using the Generalized Sucksmith-Thompson method, ${ }^{35}$ were 33.1 and $-8.1 \mathrm{kOe}$, respectively. The $R$ ratio of the sample was measured to be 0.423 . Figure $6 \mathrm{a}-\mathrm{d}$ shows the results for $\Delta H_{\mathrm{DL}}$ (a and c) and $\Delta H_{\mathrm{FL}}$ (b and $\mathrm{d}$ ) as functions of $\theta_{M}{ }^{\circ}$ at three different $I_{0}$ values: $1.0 \mathrm{~mA}$ (black squares); $1.5 \mathrm{~mA}$ (red circles); and $2.0 \mathrm{~mA}$ (blue triangles). Both conventional ( $\mathrm{a}$ and $\mathrm{b}$ ) and refined analytical methods (c and d) were used to analyze the experimental results. The results from the conventional method show incorrect divergences at $\theta_{M}{ }^{\circ}=\sim 60^{\circ}$, whereas the results from the refined method do not show this behavior over the entire $\theta_{M}{ }^{\circ}$ range. Note that both $\Delta H_{\mathrm{DL}}$ and $\Delta H_{\mathrm{FL}}$ depend on $\theta_{M}{ }^{\circ}$. The values of $\Delta H_{\mathrm{DL}}$ and $\Delta H_{\mathrm{FL}}$ should be proportional to $I_{0}$, with zero values at $I_{0}=0 .{ }^{15}$ The expectation is only satisfied for the results extracted using the refined method, as shown in Figure 6e and $\mathrm{f}$, where the results for $\Delta H_{\mathrm{DL}}$ (red circles) and $\Delta H_{\mathrm{FL}}$ (black squares) obtained at a fixed $\theta_{M}{ }^{\circ}$ value of $55^{\circ}$ are shown as functions of $I_{0}$. A large deviation from the linearity is particularly noted for the $\Delta H_{\mathrm{FL}}$ results calculated using the conventional method. Refer to Supplementary Figures S4-S6for detailed results.

\section{DISCUSSION}

The test of the conventional analytical method, which involves an analysis of the macrospin simulation results, indicates that its validity range is very limited in terms of $\theta_{M}{ }^{\circ}$ and $R$, which is primarily attributed to the singularities involved in Cramer's rule at incorrect $\theta_{M}$ ${ }^{\circ}$ values. This problem is overcome by the refined analytical method proposed in this study with detailed analytical equations, in which both the $z$ component of $\boldsymbol{H}_{\text {ext }}$ and the second-order PMA are considered. The SO effective fields that are extracted using the refined analytical method are consistent with the input SO effective fields that are used for the macrospin simulation over the entire $\theta_{M}{ }^{\circ}$ range and over a wide $R$ range of zero to two. For $R<0.85$, deviations from the input SO effective fields are $<0.4 \%$ over the entire $\theta_{M}{ }^{\circ}$ range of $0^{\circ}-82^{\circ}$ for both $\Delta H_{\mathrm{DL}}$ and $\Delta H_{\mathrm{FL}}$. Even at $R>0.85$, the deviations are $<0.8 \%$ for both $\Delta H_{\mathrm{DL}}$ and $\Delta H_{\mathrm{FL}}$ over the entire region, with the exception in some limited regions that exhibit singularities. The accuracy of the refined method is reconfirmed by an additional comparative study that involves analyzing the experimental results of harmonic Hall voltage measurements for a $\mathrm{Pt} / \mathrm{Co} / \mathrm{MgO}$ structure. An accurate analysis of the harmonic Hall voltage measurement results by the refined analytical method over very wide ranges of $\theta_{M}{ }^{\circ}$ and $R$ will significantly contribute to the identification of a dominant mechanism of the SOT and the development of highly efficient SOT devices.

\section{CONFLICT OF INTEREST}

The authors declare no conflict of interest.

\section{ACKNOWLEDGEMENTS}

This research was supported by the Creative Materials Discovery Program via the National Research Foundation of Korea (No 2015M3D1A1070465).

\section{PUBLISHER'S NOTE}

Springer Nature remains neutral with regard to jurisdictional claims in published maps and institutional affiliations.

1 Miron, I. M., Garello, K., Gaudin, G., Zermatten, P.-J., Costache, M. V., Auffret, S., Bandiera, S., Rodmacq, B., Schuhl, A. \& Gambadella, P. Perpendicular switching of a single ferromagnetic layer induced by in-plane current injection. Nature 476, 189-193 (2011).

2 Dyakonov, M. I. \& Perel, V. I. Current-induced spin orientation of electrons in semiconductors. Phys. Lett. 35A, 459-460 (1971).

3 Hirsch, J. E. Spin Hall effect. Phys. Rev. Lett. 83, 1834-1837 (1999).

4 Zhang, S. Spin Hall effect in the presence of spin diffusion. Phys. Rev. Lett. 85, 393-396 (2000).

5 Bychkov, Y. A. \& Rashba, E. I. Oscillatory effects and the magnetic susceptibility of carriers in inversion layers. J. Phys. C 17, 6039-6045 (1984).

6 Edelstein, V. M. Spin polarization of conduction electrons induced by electric current in two-dimensional asymmetric electron systems. Solid State Commun. 73, 233-235 (1990).

7 Haney, P. M., Lee, H.-W., Lee, K.-J., Manchon, A. \& Stiles, M. D. Current induced torques and interfacial spin-orbit coupling: semiclassical modeling. Phys. Rev. B 87, 174411 (2013). 
8 Kim, J., Sinha, J., Hayashi, M., Yamanouchi, M., Fukami, S., Suzuki, T., Mitani, S. \& Ohno, H. Layer thickness dependence of the current-induced effective field vector in $\mathrm{Ta} / \mathrm{CoFeB} / \mathrm{MgO}$. Nat. Mater. 12, 240-245 (2013).

9 Kurebayashi, H., Sinova, J., Fang, D., Irvine, A. C., Skinner, T. D., Wunderlich, J., Novak, V., Campion, R. P., Gallagher, B. L., Vehstedt, E. K., Zarbo, L. P., Vyborny, K., Ferguson, A. J. \& Jungwirth, T. An antidamping spin-orbit torque originating from the Berry curvature. Nat. Nanotechnol. 9, 211-217 (2014).

10 Fan, X., Celik, H., Wu, J., Ni, C., Lee, K.-J., Lorenz, V. O. \& Xiao, J. Q. Quantifying interface and bulk contributions to spin-orbit torque in magnetic bilayers. Nat. Commun. 5, 3042 (2014).

11 Liu, R. H., Lim, W. L. \& Urazhdin, S. Control of current-induced spin-orbit effects in a ferromagnetic heterostructure by electric field. Phys. Rev. B 89, 220409(R) (2014).

12 Qiu, X., Narayanapillai, K., Wu, Y., Deorani, P., Yang, D.-H., Noh, W.-S., Park, J.-H., Lee, K.-J., Lee, H.-W. \& Yang, H. Spin-orbit-torque engineering via oxygen manipulation. Nat. Nanotechnol. 10, 333-338 (2015).

13 Tatara, G. \& Kohno, H. Theory of current-driven domain wall motion: Spin transfer versus momentum transfer. Phys. Rev. Lett. 92, 086601 (2004).

14 Boulle, O., Kimling, J., Warnicke, P., Klaui, M., Rudiger, U., Malinowski, G., Swagten, H. J. M., Koopmans, B., Ulysse, C. \& Faini, G. Nonadiabatic spin transfer torque in high anisotropy magnetic nanowires with narrow domain walls. Phys. Rev. Lett. 101, 216601 (2008)

15 Van der Bijl, E. \& Duine, R. A. Current-induced torques in textured Rashba ferromagnets. Phys. Rev. B 86, 094406 (2012).

16 Wang, X. \& Manchon, A. Diffusive spin dynamics in ferromagnetic thin films with a Rashba interaction. Phys. Rev. Lett. 108, 117201 (2012).

17 Kim, K.-W., Seo, S.-M., Ryu, J., Lee, K.-J. \& Lee, H.-W. Magnetization dynamics induced by in-plane currents in ultrathin magnetic nanostructures with Rashba spinorbit coupling. Phys. Rev. B 85, 180404(R) (2012).

18 Pesin, D. A. \& MacDonald, A. H. Quantum kinetic theory of current-induced torques in Rashba ferromagnets. Phys. Rev. B 86, 014416 (2012).

19 Qiu, X., Deorani, P., Narayanapillai, K., Lee, K.-S., Lee, K.-J., Lee, H.-W. \& Yang, H. Angular and temperature dependence of current induced spin-orbit effective fields in $\mathrm{Ta} / \mathrm{CoFeB} / \mathrm{MgO}$ nanowires. Sci. Rep. 4, 4491 (2013).

20 Garello, K., Miron, I. M., Avci, C. O., Freimuth, F., Mokrousov, Y., Blugel, S., Auffret, S., Boulle, O., Gaudin, G. \& Gambardella, P. Symmetry and magnitude of spin-orbit torques in ferromagnetic heterostructures. Nat. Nanotechnol. 8, 587-593 (2013)

21 Lee, K.-S., Go, D., Manchon, A., Haney, P. M., Stiles, M. D., Lee, H.-W. \& Lee, K.-J. Angular dependence of spin-orbit spin-transfer torques. Phys. Rev. B 91, 144401 (2015).

22 Pi, U. H., Kim, K. W., Bae, J. Y., Lee, S. C., Cho, Y. J., Kim, K. S. \& Seo, S. Tilting of the spin orientation induced by Rashba effect in ferromagnetic metal layer. Appl. Phys. Lett. 97, 162507 (2010).

23 Hayashi, M., Kim, J., Yamanouchi, M. \& Ohno, H. Quantitative characterization of the spin-orbit torque using harmonic Hall voltage measurements. Phys. Rev. B 89, 144425 (2014).
24 Avci, C. O., Garello, K., Gabureac, M., Ghosh, A., Fuhrer, A., Alvarado, S. F. \& Gambardella, P. Interplay of spin-orbit torque and thermoelectric effects in ferromagnet/normal-metal bilayers. Phys. Rev. B 90, 224427 (2014).

25 Cho, S. \& Park, B.-G. Large planar Hall effect in perpendicularly magnetized W/CoFeB/ MgO structures. Curr. Appl. Phys. 15, 902-905 (2015).

26 Stillrich, H., Menk, C., Fromter, R. \& Oepen, H. P. Magnetic anisotropy and the cone state in Co/Pt multilayer films. J. Appl. Phys. 105, 07C308 (2009).

27 Shaw, J. M., Nembach, H. T., Weiler, M., Silva, T. J., Schoen, M., Sun, J. Z. \& Worledge, D. C. Perpendicular magnetic anisotropy and easy cone state in $\mathrm{Ta} / \mathrm{Co}_{60} \mathrm{Fe}_{20} \mathrm{~B}_{20} / \mathrm{MgO}$. IEEE Magn. Lett. 6, 3500404 (2015).

28 Sun, J. Z. Spin-current interaction with a monodomain magnetic body: a model study. Phys. Rev. B 62, 570 (2000).

29 Stiles, M. D., Miltat, J. in Spin Dynamics in Confined Magnetic Structures III (ed. Hillebrands B.) Ch. 5, 225-308 Springer: Berlin, (2006).

30 Cullity, B. D. in Introduction to Magnetic Materials (ed. Graham C. D.) Ch. 7 , 197-239 Wiley: Hoboken, (2008).

31 McGuire, T. R. \& Potter, R. I. Anisotropic magnetoresistance in ferromagnetic 3d alloys. IEEE Trans. Magn. Mag. 11 (4), 1018-1038 (1975).

32 Tang, H. X., Kawakami, R. K., Awschalom, D. D. \& Roukes, M. L. Giant planar Hall effect in epitaxial (Ga,Mn)As devices. Phys. Rev. Lett. 90, 107201 (2003).

33 Pai, C.-F., Nguyen, M.-H., Belvin, C., Vilela-Leao, L. H., Ralph, D. C. \& Buhrman, R. A Enhancement of perpendicular magnetic anisotropy and transmission of spin-Halleffect-induced spin currents by a $\mathrm{Hf}$ spacer layer in $\mathrm{W} / \mathrm{Hf} / \mathrm{CoFeB} / \mathrm{MgO}$ layer structures. Appl. Phys. Lett. 104, 082407 (2014).

34 Stoner, E. C. \& Wohlfarth, E. P. A mechanism of magnetic hysteresis in heterogeneous alloys. Philos. Trans. R. Soc. A 240, 599-642 (1948).

35 Sato, H., Pathak, M., Mazumdar, D., Zhang, X., Mankey, G.J., LeClair, P. \& Gupta, A Anomalous Hall effect behavior in (100) and (110) $\mathrm{CrO}_{2}$ thin films. J. Appl. Phys. 109, 103907 (2011).

This work is licensed under a Creative Commons Attribution 4.0 International License. The images or other third party material in this article are included in the article's Creative Commons license, unless indicated otherwise in the credit line; if the material is not included under the Creative Commons license, users will need to obtain permission from the license holder to reproduce the material. To view a copy of this license, visit http:// creativecommons.org/licenses/by/4.0/

(C) The Author(s) 2017

Supplementary Information accompanies the paper on the NPG Asia Materials website (http://www.nature.com/am) 